\section{G81 PARENTAL DECISION-MAKING IN HYPOPLASTIC LEFT HEART SYNDROME: THE ROLE OF A CARING DOCTOR}

doi:10.1136/archdischild-2013-304107.093

'S Bertaud, ${ }^{2} \mathrm{~S}$ Suguna Narasimhulu, ${ }^{2} \mathrm{~A}$ Ludormirsky. ${ }^{1}$ Paediatrics, Croydon University Hospital, London, UK; ${ }^{2}$ Pediatric Cardiology, NYU Langone Medical Center, New York, USA

Aims We sought to explore what parents value from their physicians when deciding on treatment for Hypoplastic Left Heart Syndrome (HLHS). We were interested in parental views on the decision-making process, the factors which influence their choices and in the level of physician involvement they desire.

Methods Eight families who had received a diagnosis of HLHS in the last 3 years and had chosen surgical intervention underwent structured interviews lasting between 1-3 hours. Patients were de-identified and results discussed with two Paediatric Cardiologists and a Professor of Bioethics to identify key themes.

Result Parental understanding of HLHS was varied; 6 of the 8 families displayed limited understanding of the long-term complications and quality-of-life issues. All remembered feelings of shock and confusion at the time of diagnosis.

When discussing what motivated their decision to choose surgery the parents all expressed ideas about the ability to give their child hope. When asked how important the following factors were in making their decision, the mean results were: (1: 'not important at all'; 5: 'very important') the doctor's opinion: 3.6 ; other people's opinion: 2.0; religion: 3.8; financial considerations: 1.9; other commitments: 2.9

Seven families felt the decision was entirely up to them. One mother instead said she, her husband and the doctors were "all together" in making the decision. Seven families said the information was presented to them in a neutral manner by the doctor. Seven families felt their doctors were optimistic about their child's outcome. Three families asked for their doctor's opinion on treatment whilst five did not, stating it was irrelevant to their decision. Seven families said parents should make decisions regarding treatment of HLHS. One father said the doctors should make the decision, whilst his partner felt it should be both parties. All eight families felt content with their decision and none expressed regrets. Conclusion Our findings offer valuable insight into the parental experience and indicate that most families with a diagnosis of HLHS felt the decision-making was entirely up to them and that information from doctors was neutral but nonetheless optimistic. Whilst parents wish to remain the final arbiters of the decision, some do value the doctor's opinion.

\section{G82 STUDY OF ACUTE LIVER FAILURE IN NEWBORNS AND YOUNG CHILDREN WITH AN UNDERLYING INHERITED METABOLIC DISEASE}

doi:10.1136/archdischild-2013-304107.094

${ }^{1}$ RM Hegarty, ${ }^{2} \mathrm{~A}$ Dhawan, ${ }^{3 P}$ Gissen. 'Neonatal Unit, Guy's and St Thomas' NHS Trust, London, UK; ${ }^{2}$ Paediatric Liver Unit, King's College NHS Trust, London, UK; ${ }^{3}$ Department of Metabolic Medicine, Great Ormond Street Hospital for Children, London, UK

Aims To study the demographic, clinical and laboratory findings, diagnoses and outcome of children under 5 years who were admitted with acute liver failure (ALF; INR > 2.0 or INR > 1.5 and encephalopathy) seconday to an underlying inherited metabolic disease (IMD).

Methods A retrospective case note review of children who were admitted between January 2001 to 2012 to a tertiary paediatric liver unit with ALF and a multi-centre review of their long term outcome. Results A total of 127 children were identified from the database. 36 children (28\%; 17 boys; median presenting age 6 weeks, range 1 day-41 months) had an underlying IMD including galactosemia in 17, mitochondrial cytopathy in 7 , ornithine transcarbamylase
(OTC) deficiency in 4, tyrosinemia type 1 in 4, Niemann-Pick C (NPC) in 3 and congenital disorder of glycosylation type 1 in 1 . The remaining aetiologies were: indeterminate in $40(32 \%)$, infectious in $15(12 \%)$, neonatal hemochromatosis in 11 (9\%), hemophagocytic syndrome in 8 (6\%), drug toxicity in $5(4 \%)$ and other in $10(8 \%)$. Of the 36 children with an IMD consanguinity was present in 16 $(44 \%)$, developmental delay in $3(8 \%)$, jaundice at presentation in 28 (78\%), hepatomegaly in $27(75 \%)$ and encephalopathy in $8(22 \%)$ The median peak (range) INR 4.8 (1.8-15), aspartate transaminase $334 \mathrm{umol} / \mathrm{L}$ (39-15791) and bilirubin 227umol/L (13-692). Liver biopsy was done in 9 children (25\%), neuroimaging in 10 children $(28 \%)$, bone marrow aspiration in $7(19 \%)$ and muscle biopsy in 5 (14\%). 29/36 children with an IMD survived (81\%). 4 children with mitochondrial cytopathy (including 1 after transplantation during the postoperative period) and 3 with NPC died. 4 children (1 OTC deficiency; 3 mitochondrial cytopathy) underwent liver transplantation. Follow up data was available for 23 children (mean follow up period, 4 years 3 months) in whom 13 (57\%) were identified as having evidence of developmental delay.

Conclusion IMD is a common cause of ALF in children. Indeterminate cases may include undiagnosed metabolic diseases. Survival of children with IMD-related ALF is good, however, long term developmental outcome is less favourable.

\section{G83 NEONATAL ECHOCARDIOGRAPHY BY NEONATOLOGISTS: EXPERIENCE SO FAR}

doi:10.1136/archdischild-2013-304107.095

'VM Shetthalli, 'S Sen, ${ }^{2} \mathrm{DG}$ Wilson, 'S Reddy. 'David Ferguson Neonatal Unit, Royal Gwent Hospital, Newport, UK; ${ }^{2}$ Department of Paediatric Cardiology, University Hospital of Wales, Cardiff, UK

Background Neonatal Echocardiography is an important investigation for assessment and clinical management of sick preterm neonate and is also essential for diagnosis of congenital heart disease. Not all neonatal intensive care units are equipped with paediatric cardiology services. Many units in UK and internationally have neonatologists doing in house echocardiograms in their units and outpatient murmur clinics. This is one of the largest study which aims to access the validity of echocardiograms done by the neonatologists compared to the cardiologists.

Methods This is a retrospective study over a $7 \frac{1}{2}$ year period in a level 3 neonatal intensive care unit. Echocardiograms were performed either in neonatal unit or outpatient murmur clinics. Selected abnormal examinations were referred to regional cardiology services. Database of all the scans were maintained electronically. This neonatal database was compared with regional paediatric cardiology database called Cardiobase ${ }^{\circledR}$ to assess concordance and discordance of the findings in referred patients.

Results A total of 2325 scans were performed on 1639 neonates from 1st September 2003 till 31st December 2011. Cardiobase ${ }^{\circledR}$ data was available for 454 scans. Concordance was found in $91.8 \%$ of these scans and partial concordance was found in further $4.4 \%$ of scans. 31 infants $(1.6 \%$ of total infants scanned) had critical structural abnormalities and all of these were identified correctly. $3.7 \%$ of the scans had discordant findings and most of these were false positives and they were referred to exclude critical events. Six scans had had false negative findings. Sensitivity and specificity for the scans done by neonatologists was $98 \%$.

Abstract G83 Table 1 Concordance rates

\begin{tabular}{lll}
\hline Cardiobase data (Total) & $\mathbf{4 5 4}$ scans & $\mathbf{1 0 0 \%}$ \\
\hline Concordance & 417 scans & $91.8 \%$ \\
Partial Concordance & 20 scans & $4.4 \%$ \\
Discordance & 17 scans & $3.7 \%$ \\
\hline
\end{tabular}

